\title{
Localization in silicon nanophotonic slow-light waveguides
}

\author{
SHAYAN MOOKHERJEA ${ }^{1 *}$, JUNG S. PARK ${ }^{1}$, SHUN-HUI YANG ${ }^{2}$ AND PRABHAKAR R. BANDARU² \\ 'University of California, San Diego, Electrical Engineering, Mail Code 0407, La Jolla, California 92093-0407, USA \\ 2University of California, San Diego, Mechanical and Aerospace Engineering, Mail Code 0411, La Jolla, California 92093-0411, USA \\ *e-mail: mookherjea@ece.ucsd.edu
}

Published online: 27 January 2008; doi:10.1038/nphoton.2007.278

Slowing down light on a chip can lead to the development of optical buffers $^{1}$, filters ${ }^{2,3}$ and memory elements ${ }^{4}$ useful for optical interconnects and for resonantly enhanced chip-based nonlinear optics ${ }^{5,6}$. Several approaches to slow light rely on the phenomenon of light interference in a sequence of coupled resonators ${ }^{1-4,7-11}$; however, light interference is also responsible, in disordered structures, for the localization of light, an effect particularly prominent in one-dimensional devices $^{12,13}$. Until now, the length of the waveguides investigated has been less than the localization length. Here we report the first observation of light localization in compact silicon nanophotonic slow-light waveguides consisting of long sequences of coupled resonators. Our results show that disorder limits how much light can be slowed, and that localization leads to spatially concentrated and locally trapped light in a quasi-one-dimensional waveguide at wavelengths near the band edge.

Optical slow-wave structures, like their microwave counterparts $^{14}$, consist of a chain or network of repeated unit cells in which light propagates by tunnelling from one unit cell to its nearest neighbours ${ }^{2}$. Each unit cell could consist, for example, of a microring resonator ${ }^{1,3}$, a defect resonator in a photonic $\operatorname{crystal}^{10,15}$, or a microsphere ${ }^{16,17}$. This underlying physical principle of nearest-neighbour coupling can be used to derive an analytical description of the waveguide dispersion, similar to the tight-binding theory used in solid-state physics $^{8,18}$. Another model uses matrices to describe the interactions between adjacent resonators ${ }^{19}$, or the entire slowwave structure ${ }^{20}$ and this model is especially well suited to understanding the effects of disorder.

In computing the fields in a slow-wave structure, which consists of a concatenation of unit cells, the field amplitude is described by a column vector $\mathbf{u}$, which lists the fields in the individual unit cells that comprise the structure. The evolution of $\mathbf{u}$ is described by a matrix equation $i \mathrm{~d} \mathbf{u} / \mathrm{d} t=M \mathbf{u}$, where the coupling matrix $M$ is typically band tri-diagonal in form, because the field in each unit cell couples only to the fields in its nearest neighbours. Consequently, the dispersion relationship has the familiar tightbinding form ${ }^{8}, \omega / \Omega=1+2 \kappa \cos (K R)$, where $\omega$ is the frequency, $\kappa$ is the coupling coefficient, $R$ is the centre-to-centre distance between unit cells, $K$ is the Bloch wavenumber and $\Delta \omega \equiv 2 k \Omega$ is the half-width of the waveguiding band around the centre frequency $\Omega$ (see Supplementary Information). Slow light is particularly observed near the band edges $\Omega \pm \Delta \omega$ (see Fig. 1a), where the group velocity, defined as the slope of the dispersion curve $v_{\mathrm{g}} \equiv \mathrm{d} \omega / \mathrm{d} K$, goes to zero. However, in practice, the unit cells cannot be exactly identical because of fabrication tolerances. As shown in the inset to Fig. 1a, a closer examination of the edge shows the existence of a band tail, and the slope of the dispersion curve (and hence $v_{\mathrm{g}}$ ) is no longer zero ${ }^{21}$.

To understand why the group velocity does not go to zero, it is necessary to consider the connection between group velocity and the density of states. In a weakly disordered one-dimensional slow-wave structure consisting of $N$ unit cells with periodicity $R$, the group velocity $v_{\mathrm{g}}$ is inversely proportional to the density of states, and is given by ${ }^{21}$

$$
v_{\mathrm{g}}=\frac{1}{\hat{\rho}(\omega)} \frac{1}{2 \pi / R}\left(1+\frac{1}{N R} \frac{\mathrm{d} \phi}{\mathrm{d} k}\right)
$$

where $\hat{\rho}$ is the optical density of states (normalized to the unit integral over the waveguide band as indicated by ${ }^{\wedge}$ ), and $\phi$ is the disorder-induced (scattering) phase shift. In a perfectly ordered structure, $\hat{\rho}\left(\omega_{\text {edge }}\right) \rightarrow \infty$ at the band edges ${ }^{22}$ and thus $v_{\mathrm{g}} \rightarrow 0$, whereas, as shown in Fig. $1 b$, in a disordered structure, $\hat{\rho}\left(\omega_{\text {edge }}\right)$ only reaches a certain maximum value that depends on the statistics of variation of the elements of $M-$ most importantly, the mean and standard deviation of the distribution of coupling coefficients, $\kappa$ and $\delta \kappa$, respectively ${ }^{21}$. The slowing factor is (see Supplementary Information)

$$
S \equiv \frac{c}{v_{\mathrm{g}} \text { at band edge }}=\frac{\lambda}{R} \frac{1}{\left(\delta \kappa^{2} \cdot \kappa\right)^{1 / 3}},
$$

where $c$ is the speed of light in a vacuum. Equation (2) shows that $S$ $\rightarrow \infty$ (because $v_{\mathrm{g}}$ at the band edge $\rightarrow 0$ ) if $\delta \kappa \rightarrow 0$. However, for a typical structure, if $\kappa=10^{-2}$ (see Supplementary Information), $\delta \kappa=5 \%$ of $\kappa$, and $R=10 \lambda$, then $S=74$, a much more modest slowing factor. Experimental observations also indicate $S \approx$ $10-100($ refs 1,3$)$.

If we examine the field distributions corresponding to the band tail in the dispersion relationship, we see that those field distributions are localized by disorder, as shown in Fig. 2. From such profiles, a localization length may be defined as the root-mean-squared width of the intensity distribution for the localized modes. From Monte Carlo simulations of such 

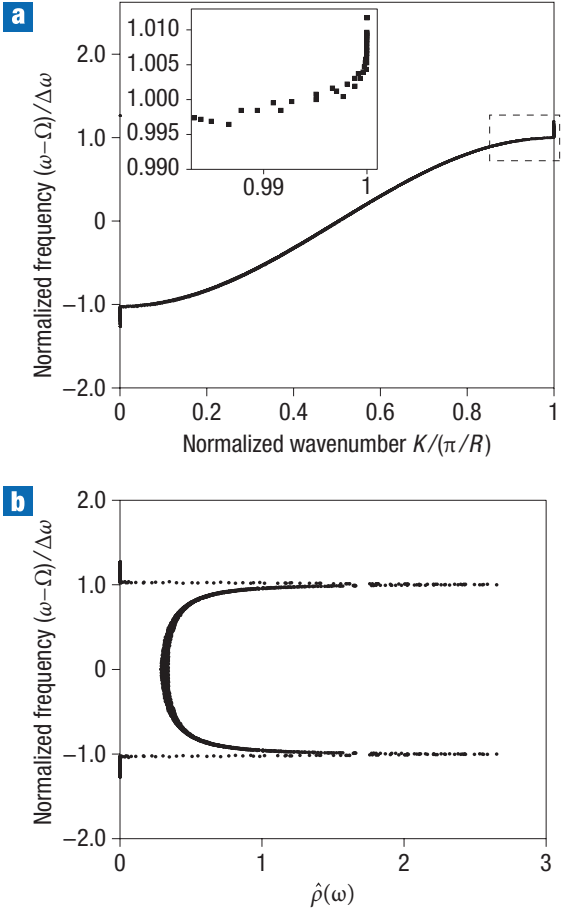

Figure 1 Dispersion and density of states for a weakly disordered slow-wave structure. a, Dispersion relationship calculated $a b$ initio $^{21}$ for a slow-wave structure with $1 \%$ disorder in the nearest-neighbour coupling coefficient. For such weak disorder, the dispersion follows the tight-binding (cosine) function almost exactly, except at the band edge, shown by the inset. The band-edge tail shows that $v_{\mathrm{g}} \equiv \mathrm{d} \omega / \mathrm{d} K$ is non-zero. $\mathbf{b}$, Over the same range of frequencies, the density of states is calculated, showing that the density of states $\hat{\rho}(\omega)$ does not diverge to infinity at the band edges in the presence of disorder, as it should in the ideal model ${ }^{22}$.

profiles, we observe, as shown in Fig. $2 \mathrm{~d}$, that the localization length decreases exponentially with increasing disorder but has a finite non-zero lower limit, given by the offset of the exponential fit, which was calculated to be 5.6 unit cells for a finite-length structure consisting of a chain of 100 coupled resonators. Notice also that, even for small changes in $\omega$ in the farthest part of the tail, the location of the localized modal field patterns in the chain can vary-an observation that we shall refer to later. Devices fabricated so far have consisted of only a few unit cells, because of lithographic challenges in patterning long chains of identical optical resonators, and if the localization length exceeds the device length, light propagates from input to output without clear evidence of localization. However, because, formally, all the eigenmodes are localized for any value of disorder in one-dimensional waveguide structures ${ }^{23}$, localization is indeed possible in sufficiently long chains, which are necessary for practical applications as optical interconnects ${ }^{4}$.

To observe localization effects, long slow-wave structures consisting of a large number of coupled resonators are needed, as well as the use of a material system with a high index contrast between core and cladding, such as the 100-microring chain demonstrated recently ${ }^{1}$. In silicon-on-insulator (SOI) photonics, the absence of a practical optical gain mechanism for $1,550-\mathrm{nm}$ light suggests the use of a more compact form of resonator for this study, and one with higher light throughput,
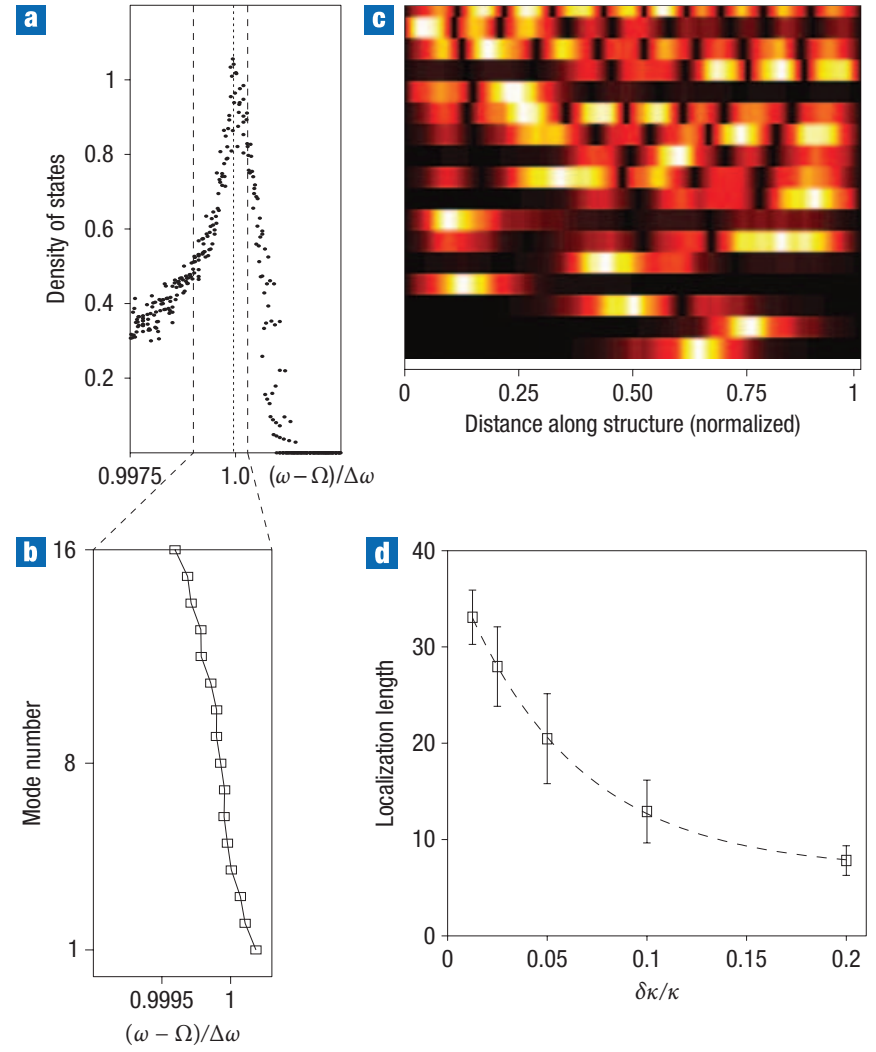

Figure 2 Spectral distribution of localization. a, The density of states near the band edge calculated for a slow-wave structure. The results of 32 Monte Carlo simulations are shown. b. Eigenfrequencies from a single Monte Carlo calculation are labelled from the band edge inwards $(1=$ farthest detuning from band centre), showing that disorder creates states beyond the band edge of the ideal structure. c, The corresponding field distributions along the (normalized) length of the slow-wave structure, showing that field distributions for frequencies near the band edge, at the bottom of the figure, are localized, and those inside the band, towards the top of the figure, are extended. d, The minimum localization length (in unit cells) for a finite-length structure consisting of 100 coupled resonators, as a function of the disorder, $\delta \kappa / \kappa$. The points are calculated from Monte Carlo simulations, and the dashed line is an exponential fit, from which can be inferred a minimum localization length of six unit cells.

because it is not necessary to use very high quality factors to create a coupled-resonator slow-wave waveguide. Furthermore, it is difficult in practice to measure a transfer function resembling the theoretical ideal in long sequences of coupled microrings, because each unit cell (a single microring of diameter $D$ ) typically supports a large number of modes of the order of $\sim \pi D / \lambda$ which can be coupled by very weak imperfections; the transmission spectrum shows many spurious ripples ${ }^{1}$ and the resultant bandstructure is highly complicated. Our structure is shown in Fig. 3 and consists of a sequence of cuboidal resonators (with rounded edges to reduce scattering), which load a single-mode optical waveguide. The volume of each resonator is $\sim 1.1 \mu \mathrm{m}^{3}$. We calculate, using a threedimensional vectorial plane-wave expansion (PWE) algorithm, that a slow-wave mode of this structure has a band edge in the vicinity of $1,575 \mathrm{~nm}$, as shown in Fig. $4 \mathrm{a}$, and there are no other propagating slow-wave modes in a window of $\pm 5 \mathrm{~nm}$ around 

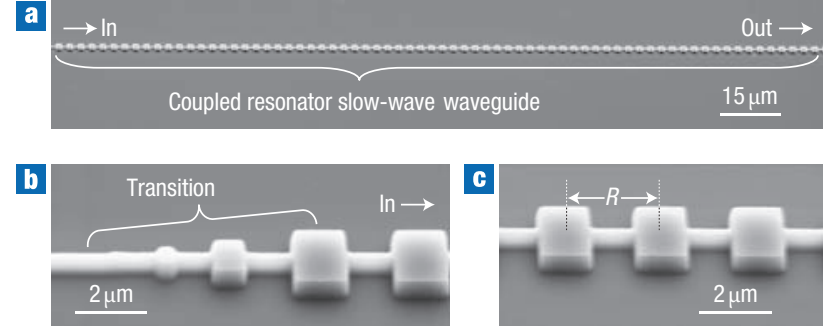

Figure 3 Slow-wave coupled-resonator waveguides. a, The fabricated structure on an SOI chip consists of a single-mode waveguide loaded periodically with 100 resonators (about 70 resonators are shown here), with two transition sections to better match impedances between the input/output sections and the slow-wave section. The top and side claddings are air, and the bottom cladding is silicon dioxide. $\mathbf{b}$, A magnified view of the transition section. $\mathbf{c}$, A magnified view of the slow-wave section, with the periodicity $R$ being $2.75 \mu \mathrm{m}$.

this wavelength. In the absence of disorder, we would expect to see a 'frozen light' mode spanning the entire length of the structure, similar to the in-phase supermode of a waveguide array. In contrast, we would expect disorder, unavoidable in the fabrication process, to create localized states in this region, with a localization length that increases as the input optical wavelength is increased, that is, moving inside the band, away from the edge.

The photonic slow-wave structures were fabricated on an SOI wafer using electron-beam lithography and dry-etching techniques (see Methods). The photonic-wire waveguides were single-moded and had a modal cross-section of $0.25 \mu \mathrm{m} \times 0.5 \mu \mathrm{m}$, and the resonators had dimensions of $1.5 \mu \mathrm{m} \times 1.5 \mu \mathrm{m} \times 0.5 \mu \mathrm{m}$, with $R=2.75 \mu \mathrm{m}$ along the waveguide axis. On excitation with a tunable continuous-wave (c.w.) laser, field profiles as shown in Fig. 4d were recorded at wavelengths of interest near the band edge. The measurements shown in Fig. 4 were performed on devices with 100 resonators in the slow-wave section (as shown in Fig. 3a). An estimate of the number of unit cells $N^{*}$ needed to observe localization without encountering boundary effects is given by $^{24} N^{\star}=9(\kappa / \delta \kappa)^{2 / 3}$, which evaluates to 93 unit cells for $3 \%$ disorder and 66 unit cells for $5 \%$ disorder. (Higher values of disorder may not obey the ballistic propagation model in this waveguide geometry.) In fact, structures with 5 or 50 resonators did not reveal evidence of localization, showing that longer slow-wave structures are necessary to observe localization in slow-light structures, even with the high index contrast of the SOI material system.

The band structure shown in Fig. 4a was calculated using the PWE method-the blue dots (without squares) are numerical artefact. The field profiles corresponding to the blue dots are found to contain spurious transverse resonances spanning the whole width of the calculation cell, because the PWE algorithm imposes periodic boundary conditions along all the cellular boundaries. These mathematical solutions do not correspond to physically realistic fields and are ignored.

At the band-edge wavelengths predicted by Fig. 4a, the transmission spectrum decreases rapidly, as shown in Fig. 4b. Some fluctuation in the measured power is seen in this regime, indicated by the shaded blue region, which is thicker around the band-edge transition (for example, at $1,576 \mathrm{~nm}$ compared with $1,576.5 \mathrm{~nm}$ or longer wavelengths). Although not fully studied at this time, this observation agrees with the theoretical prediction
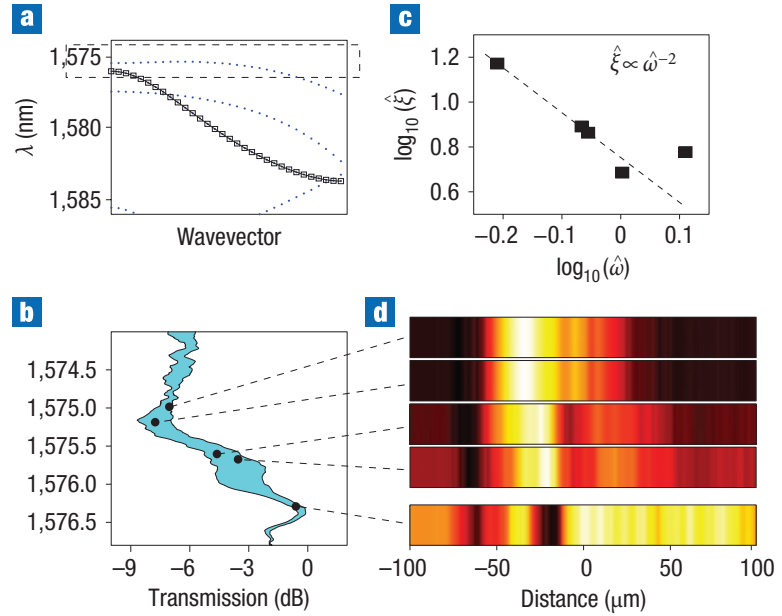

Figure 4 Experimental measurements of localization. a, Band structure calculated using a vectorial three-dimensional plane-wave method, showing the coupled-resonator slow-wave band (squares). Blue dots are non-physical solutions explained in the text. $\mathbf{b}$, Measured transmission spectrum for the band-edge wavelengths indicated by the dashed box in $\mathbf{a}$. The maximum-minimum range of measurements over repeated scans, shown in blue, is discussed in the text. c, Log-log plot of the localization length $\hat{\xi}$ (normalized units) versus frequency $\hat{\omega}$ (normalized units), in agreement with the theoretically calculated shape (ref. 23 , Fig. 8.8). d, Measured spatial profiles of a representative extended field distribution at $1,576.16 \mathrm{~nm}$, and localized field distributions at $1,575.64 \mathrm{~nm}, 1,575.60 \mathrm{~nm}$, $1,575.10 \mathrm{~nm}$ and $1,575.00 \mathrm{~nm}$ (from bottom to top).

from Fig. 2 that small changes in $\omega$ can lead to large changes in the spatial pattern of localized fields, which affects the transmission in finite-length structures. This spectral region, which lies between the localized and extended modes, could possibly support 'necklace' modes ${ }^{25}$, which are multiresonance states with fast temporal behaviour. The presence of a small peak $(\sim 1.5 \mathrm{~dB})$ in the transmission at the band edge has also been recently observed in a different slow-wave structure ${ }^{26}$ and is conjectured to be related to enhanced coupling to the slow-light mode.

The normalized localization length is calculated from the field profiles by numerically finding the root-mean-squared width of the intensity distribution, and dividing by the unit cell length. Figure $4 \mathrm{c}$ plots the localization length $(\hat{\xi})$ versus frequency $(\hat{\omega})$, both in normalized units, for the localized profiles shown in Fig. $4 \mathrm{~d}$. The figure shows that $\hat{\xi} \propto \hat{\omega}^{-2}$-that is, it is a straight line with slope -2 on a $\log -\log \operatorname{plot}^{27}$, near the band-edge, $\hat{\omega} \in(-0.2,0)$-and is flat for positive detuning from the frequency of maximum localization, in agreement with the theoretically predicted behaviour of localization ${ }^{23}$ calculated using a Green's function formalism with the coherent potential approximation. Based on simulations, we predict in Fig. $2 \mathrm{~d}$ that for a finite-length structure consisting of a chain of 100 coupled resonators, the localization length should be approximately 5.6 unit cells. By way of comparison, from Fig. 4c, the smallest localization length measured was 6 unit cells $(17 \mu \mathrm{m})$.

In conclusion, we have shown the presence of localization in nanophotonic SOI structures. Optical slow-wave structures, such as coupled photonic-crystal cavities, coupled microrings, coupled Fabry-Pérot cavities, and so on, are promising 'engineered dispersion' slow-light waveguides, which can slow light significantly in a small device footprint because they can be lithographically patterned or self-assembled on length scales 
comparable to the optical wavelength. However, this regime is particularly susceptible to localization, as has been theoretically predicted and as we have shown experimentally. Unlike in the perfectly ordered theoretical model, practical structures (and those models that do take into account the effects of disorder ${ }^{21}$ ) do not exhibit a divergence in the density of states at the band edge. Consequently, the zero-group-velocity light distribution predicted for the ordered structure is not sustained, and applications of slow-light devices that rely on this feature are not robust to real-world fabrication tolerances ${ }^{28,29}$. Practical devices display the intertwined effects of order and disorder, manifest particularly in localization near the band edge. Future work will involve a study of dynamic field confinement and the orderdisorder transition, possibly controllable in SOI structures by electrically induced optical modulation. Also of interest are the coupled-resonator structures with true bandgap characteristics, such as coupled cavities in photonic crystals. Such structures will enable research in a new generation of photonic devices that combine engineered functionality with naturally occurring phenomena such as localization.

\section{METHODS}

\section{FABRICATION}

The structures shown in Fig. 3 were prepared on an SOI wafer, with $0.5-\mu \mathrm{m}$ silicon layer and $1-\mu \mathrm{m}$ buried oxide. Electron-beam resist poly(methyl methacrylate) (PMMA) $495 \mathrm{~K} 4 \%$ in chlorobenzene was spin-coated at 3,000 r.p.m. to a thickness of $280 \mathrm{~nm}$, and baked at $180^{\circ} \mathrm{C}$ for $5 \mathrm{~min}$. Patterns were exposed using a Raith50 electron-beam lithography instrument, followed by development in methyl isobutyl ketone:isopropyl alcohol (MIBK:IPA) (1:3) for $1 \mathrm{~min}$. Nickel was evaporated onto the pattern to a thickness of $35 \mathrm{~nm}$ using a Temescal BJD 1800 electron-beam evaporator. Acetone was used to lift off the PMMA, leaving the nickel mask for the following etching step.

First, an oxygen plasma descum step was performed (200 W radiofrequency, r.f.) power, 30 mtorr pressure, $10 \mathrm{~min}$ ), followed by a reactive ion etch using a mixture of $\mathrm{BCl}_{3}(10$ s.c.c.m.) and $\mathrm{Ar}(10$ s.c.c.m.) at $100 \mathrm{~W}$ r.f. power and 30 mtorr pressure for a total of $10 \mathrm{~min}$. The nickel was removed by immersing the chip in Nickel Etchant (Transene, Type TFB) for $10 \mathrm{~s}$. The chip was lapped down to a thickness of $200 \mu \mathrm{m}$, cleaved and mounted on a sample holder for measurement

\section{MEASUREMENT}

Coupling into and out of the waveguides was achieved using tapered and lensed polarization-maintaining fibres (Oz-Optics), aligned to the chip using six-axis micrometer stages (Newport Ultralign) equipped with differential micrometers. A C $+\mathrm{L}$ band mode-hop-free tunable c.w. laser (Agilent 81640A) was used as the input source with a typical input power of $100 \mu \mathrm{W}$. The spectrum (Fig. 4b) was recorded by sweeping the laser wavelength and monitoring the output using an InGaAs photodiode, while simultaneously recording the wavelength.

To record the spatial distribution of the field (Fig. 4d), a modified knifeedge method was used, a simple and robust method that is insensitive to misalignment, knife edge diffraction and geometric aperturing ${ }^{30}$. The device plane of the chip (as shown in Fig. 3a) was confocally imaged using a microscope (Olympus BX series, with Mitutoyo M-Plan-APO NIR objective) focused onto a highly sensitive InGaAs photoreceiver (New Focus Femtowatt 2153). The magnification was chosen so that the field of view at the detector images the slow-wave section, and not the input/output fibres or chip facets. The laser source was set to the particular wavelength of interest and was modulated at a frequency less than $750 \mathrm{~Hz}$, and the photoreceiver output was measured by a lock-in amplifier (Stanford Research Systems SR830). As the knife edge was scanned across the field of view at a constant speed (using a Newport ILS translation stage and ESP300 motion controller), the resultant trace was recorded. The field profiles were obtained from the measured traces by smoothing, using a moving average filter with a window of $250 \mathrm{~nm}$, which is not more than the calibrated precision of the linear stage, and differentiating the resultant trace. To factor out the coupling and background absorption features, all traces were normalized by dividing each by the same 'control' trace, measured at a wavelength at which the field was extended. It is implicitly assumed that the coupling and background loss coefficient do not vary over the 2-nm spectral window of interest, which is substantially narrower than the narrowest bandwidth of any optical elements in the measurement set-up. When measured in this way, traces for localized fields show broad dark regions (local normalized field intensity $\ll 1$ ) compared with extended fields at the same input optical power levels.

Received 6 September 2007; accepted 28 November 2007; published 27 January 2008

\section{References}

1. Xia, F., Sekaric, L. \& Vlasov, Y. Ultracompact optical buffers on a silicon chip. Nature Photonics 1 , 65-71 (2007).

2. Melloni, A., Morichetti, F. \& Martinelli, M. Optical slow wave structures. Optics and Photonics News $14,44-48(2003)$

3. Poon, J. K. S., Zhu, L., DeRose, G. \& Yariv, A. Transmission and group delay of microring coupledresonator optical waveguides. Opt. Lett. 31, 456-458 (2006).

4. Khurgin, J. B. Optical buffers based on slow light in electromagnetically induced transparent media and coupled resonator structures: comparative analysis. J. Opt. Soc. Am. B 22, 1062-1074 (2005).

5. Hill, M. T. et al. A fast low-power optical memory based on coupled micro-ring lasers. Nature 432, $206-209$ (2004)

6. Heebner, J. E., Chak, P., Pereira, S., Sipe, J. E. \& Boyd, R. W. Distributed and localized feedback in microresonator sequences for linear and nonlinear optics. J. Opt. Soc. Am. B 21, 1818-1832 (2004).

7. Stefanou, N. \& Modinos, A. Impurity bands in photonic insulators. Phys. Rev. B 57, $12127-12133(1998)$

8. Yariv, A., Xu, Y., Lee, R. K. \& Scherer, A. Coupled-resonator optical waveguide: a proposal and analysis. Opt. Lett. 24, 711-713 (1999).

9. Bayer, M. et al. Optical demonstration of a crystal band structure formation. Phys. Rev. Lett. 83, 5374-5377 (1999).

10. Olivier, S. et al. Miniband transmission in a photonic crystal coupled-resonator optical waveguide. Opt. Lett. 26, 1019-1021 (2001)

11. Mookherjea, S. \& Yariv, A. Coupled resonator optical waveguides. IEEE J. Sel. Top. Quant. Electron. 8, $448-456$ (2002).

12. Mott, N. F. \& Twose, W. D. The theory of impurity conduction. Adv. Phys. 10, 107-163 (1961).

13. John, S., Sompolinksy, H. \& Stephen, M. J. Localization in a disordered elastic medium near two dimensions. Phys. Rev. B 27, 5592-5603 (1983)

14. Slater, J. C. Microwave Electronics (Dover, New York, 1969).

15. Happ, T. D., Kamp, M., Forchel, A., Gentner, J.-L. \& Goldstein, L. Two-dimensional photonic crystal coupled-defect laser diode. Appl. Phys. Lett. 82, 4-6 (2003).

16. Möller, B. M., Artemyev, M. V. \& Woggon, U. Coupled-resonator optical waveguides doped with nanocrystals. Opt. Lett. 30, 2116-2118 (2005).

17. Astratov, V. N., Franchak, J. P. \& Ashili, S. P. Optical coupling and transport phenomena in chains of spherical dielectric microresonators with size disorder. Appl. Phys. Lett. 85, 5508-5510 (2004).

18. Bayindir, M.. Temelkuran, B. \& Ozbay, E. Tight-binding description of the coupled defect modes in three-dimensional photonic crystals. Phys. Rev. Lett. 84, 2140-2143 (2000).

19. Poon, J. K. S. et al. Matrix analysis of microring coupled-resonator optical waveguides. Opt. Express 12, 90-103 (2004).

20. Mookherjea, S. Spectral characteristics of coupled resonators. J. Opt. Soc. Am. B 23, $1137-1145$ (2006)

21. Mookherjea, S. \& Oh, A. Effect of disorder on slow light velocity in optical slow-wave structures Opt. Lett. 32, 289-291 (2007)

22. Economou, E. N. Green's Functions in Quantum Physics 3rd edn (Springer, Berlin, 2006).

23. Sheng, P. Introduction to Wave Scattering, Localization and Mesoscopic Phenomena (Academic Press, San Diego, 1995).

24. Malyshev, V. \& Moreno, P. Hidden structure of the low-energy spectrum of a one-dimensional localized Frenkel exciton. Phys. Rev. B 51, 14587-14593 (1995).

25. Bertolotti, J., Gottardo, S., Wiersma, D., Ghulinyan, M. \& Pavesi, L. Optical necklace states in Anderson localized ID systems. Phys. Rev. Lett. 94, 113903 (2005).

26. Volkov, V. S., Bozhevolnyi, S. I., Frandsen, L. H. \& Kristensen, M. Direct observation of surface mode excitation and slow light coupling in photonic crystal waveguides. Nano Lett. 7, 2341-2345 (2007).

27. Theodorou, G. \& Cohen, M. C. Extended states in a one-dimensional system with off-diagonal disorder. Phys. Rev. B 13, 4597-4601 (1976).

28. Yanik, M. F. \& Fan, S. Stopping light all optically. Phys. Rev. Lett. 92, 083901 (2004).

29. Soljačíc, M. et al. Photonic crystal slow light enhancement of nonlinear phase sensitivity. J. Opt. Soc. Am. B 19, 2052-2059 (2002).

30. Firester, A. H., Heller, M. E. \& Sheng, P. Knife-edge scanning measurements of sub-wavelength focused light beams. Appl. Opt. 16, 1971 (1977).

\section{Acknowledgements}

This work was supported by the US National Science Foundation under grants ECCS-0642603 and ECCS-0403589. J.P acknowledges sponsorship support provided by the National Science Graduate Student Fellowship. The authors are grateful to the San Diego Supercomputer Center for

computational resources, and to Y. Fainman for making his lapping machine available to us. A. Oh provided assistance with numerical simulations.

Correspondence and requests for materials should be addressed to S.M.

Supplementary information accompanies this paper on www.nature.com/naturephotonics.

\section{Author contributions}

S.M. contributed to all aspects of the project. J.S.P. contributed to experimental work and data analysis. S.H.Y. and P.B. contributed materials. All authors contributed to discussing the results and writing the paper.

Reprints and permission information is available online at http://npg.nature.com/reprintsandpermissions 